\title{
GM-CSF-loaded chitosan hydrogel as an immunoadjuvant enhances antigen-specific immune responses with reduced toxicity
}

\author{
Kyung Hee Noh ${ }^{1,2 \dagger}$, Yeong Min Park ${ }^{3 \dagger}$, Hyuk Soon Kim ${ }^{4 \dagger}$, Tae Heung Kang ${ }^{3}$, Kwon-Ho Song ${ }^{1,2}$, Young-Ho Lee ${ }^{1,2}$, \\ Yeongseon Byeon ${ }^{3}$, Hat Nim Jeon ${ }^{3}$, In Duk Jung ${ }^{3}$, Byung Cheol Shin ${ }^{5}$, Kyung-Mi Lee ${ }^{2}$, Seung-Yong Seong ${ }^{6,7}$, \\ Hee Dong $\mathrm{Han}^{3^{*}}$ and Tae Woo Kim ${ }^{1,2^{*}}$
}

\begin{abstract}
Background: The application of vaccine adjuvants has been vigorously studied for a diverse range of diseases in order to improve immune responses and reduce toxicity. However, most adjuvants have limited uses in clinical practice due to their toxicity.

Methods: Therefore, to reduce health risks associated with the use of such adjuvants, we developed an advanced non-toxic adjuvant utilizing biodegradable chitosan hydrogel (CH-HG) containing ovalbumin (OVA) and granulocyte-macrophage colony-stimulating factor (GM-CSF) as a local antigen delivery system.

Results: After subcutaneous injection into mice, OVA/GM-CSF-loaded CH-HG demonstrated improved safety and enhanced OVA-specific antibody production compared to oil-based adjuvants such as Complete Freund's adjuvant (CFA) or Incomplete Freund's adjuvant (IFA). Moreover, CH-HG system-mediated immune responses was characterized by increased number of OVA-specific CD4 ${ }^{+}$and $\mathrm{CD}^{+} \mathrm{INF}^{+} \gamma^{+} \mathrm{T}$ cells, leading to enhanced humoral and cellular immunity.
\end{abstract}

Conclusions: In this study, the improved safety and enhanced immune response characteristics of our novel adjuvant system suggest the possibility of the extended use of adjuvants in clinical practice with reduced apprehension about toxic side effects.

Keywords: Adjuvant, Chitosan, Hydrogel, Immune response

\section{Background}

Vaccine adjuvants have been extensively studied in order to enhance their safety and improve the immune responses elicited by the accompanying antigen for immunotherapy [1-3]. However, conventional adjuvants evoke serious side effects due their toxicity [4]. This has limited their use in clinical trials in recent years and poses as a serious hurdle to effective adjuvant-based immunotherapy, resulting in side effects such as boil and pyrexia occurring in clinical trials. Mineral oil-based media, Complete Freund's adjuvant (CFA) and Incomplete

\footnotetext{
* Correspondence: hanhd@kku.ac.kr; twkim0421@korea.ac.kr

${ }^{\dagger}$ Equal contributors

${ }^{3}$ Department of Immunology, School of Medicine, Konkuk University, 268 Chungwondaero, Chungju-Si, Chungcheongbuk-Do 380-701, South Korea 'Laboratory of Infection and Immunology, Graduate School of Medicine, Korea University, Gojan-1 Dong, Ansan-Si, Gyeonggi-Do 425-707, South Korea Full list of author information is available at the end of the article
}

Freund's adjuvant (IFA) [5,6], are available as commercialized products and are potent adjuvants commonly used in animal research, as they can augment both humoral and cellular immune responses to a wide range of antigens. However, they are toxic to human subjects, and their use in animals are now also discouraged or banned by many institutional animal ethics committees due to their noxious side effects [4]. These limitations enforce the need for novel and improved adjuvant systems to replace the existing ones.

Here, we have developed an improved chitosan hydrogel $(\mathrm{CH}-\mathrm{HG})$ system as a vaccine adjuvant to enhance induced immune responses and reduce toxicity $[7,8]$. Chitosan is particularly attractive for clinical and biological applications due to its low toxicity, low immunogenicity, biocompatibility, and biodegradability $[8,9]$. In addition, $\mathrm{CH}-\mathrm{HG}$ displays a liquid-solid phase transition 
depending on temperature, allowing $\mathrm{CH}-\mathrm{HG}$ to simply be injected at the diseased site without a requirement for surgical procedure. Therefore, $\mathrm{CH}-\mathrm{HG}$ may lead to enhanced safety and immune responses at disease sites. Moreover, $\mathrm{CH}-\mathrm{HG}$ can be gradually degraded by enzymes in the body after the antigen has been completely released.

Granulocyte-macrophage colony-stimulating factor (GM-CSF) has been used as a vaccine adjuvant, which stimulates macrophage differentiation and proliferation, and to activate antigen presenting cells (APCs) such as dendritic cells (DCs) or macrophages. These qualities make GM-CSF important and relevant to developing vaccine therapies [10-12]. GM-CSF can augment the primary immune response of murine spleen cells to sheep red blood cells and increase interleukin-1 secretion [13]. In addition, GM-CSF can increase the immunogenicity of tumors in animal models and stimulate both humoral and cellular immune responses, thus serving as an effective adjuvant for DNA or peptide based vaccines $[14,15]$. This increased immune response is presumed to be a result of the GM-CSF-mediated increase in maturation and function of APCs [16]. Further studies, using microspheres of encapsulated GM-CSF mixed with irradiated tumor cells that were injected subcutaneously also demonstrated increased tumor immunogenicity [17]. These studies motivated us to ask whether GM-CSF function could be improved in prolonged immune responses at a local adjuvant site. Here, we present a novel immunization strategy using $\mathrm{CH}$-HG-loaded GM-CSF/OVA as an adjuvant system to increase immunogenicity in both humoral and cellular immune responses in a mouse model as a proofof-concept, and in order to approach a clinically relevant study of GM-CSF in CH-HG as an enhanced adjuvant for vaccination that evokes an improved immune response, while exhibiting lower toxicity compared to existing adjuvants.

\section{Methods}

Mice

Female C57BL/6 mice (6 weeks old, 20 g) were purchased from Daehan Biolink (Chungbuk, Korea) and maintained under a protocol approved by the Korea University Institutional Animal Care and Use Committee (KUIACUC-2013-210). All procedures were performed in accordance with recommendations for the proper animal care and use.

\section{Preparation of OVA and GM-CSF-loaded CH-HG}

We have previously reported the physical characteristics of $\mathrm{CH}-\mathrm{HG}$ as an in vitro or in vivo depot system after subcutaneous (SC) injection [8,9]. We prepared $\mathrm{CH}-\mathrm{HG}$ containing OVA and/or GM-CSF with mild modification made to our previously reported method. Briefly, chitosan solution (medium molecular weight of $161 \mathrm{kDa}$, viscosity of 200,000 cps and a degree of deacetylation of $80 \%$ ) was obtained by dissolving $40 \mathrm{mg}$ of chitosan in $1.8 \mathrm{ml}$ of 0.1 $\mathrm{M} \mathrm{HCl}$ solution. Glycerol 2-phosphate disodium salt hydrate $(\beta-\mathrm{GP})$ solution containing $50 \mu \mathrm{g}$ of OVA and/or 50 ng of GM-CSF was prepared by dissolving $0.2 \mathrm{~g}$ of $\beta$-GP and predetermined amount of OVA or GM-CSF in $0.2 \mathrm{ml}$ of distilled water. The $\mathrm{CH}$ solution was cooled to $4^{\circ} \mathrm{C}$ and continuously stirred while adding $0.2 \mathrm{ml}$ of mixed solution was added. The final product is successfully formed hydrogel at body temperature and physiological $\mathrm{pH}$ in vivo after subcutaneous (SC) injection into mice. Notably, the mice did not suffer severe side effects, including pus formation or inflammation, and maintained a healthy appearance after CH-HG implantation.

\section{Safety test of CH-HG in mice after SC injection}

To confirm the safety of adjuvants, $50 \mu \mathrm{l}$ of $\mathrm{CH}-\mathrm{HG}$, Complete Freund's adjuvant (CFA), or Incomplete Freund's adjuvant (IFA) were injected subcutaneously into mice. The external morphologies of the adjuvants were monitored in the mice, and the hydrogel volume was measured using calipers for 14 days. After 14 days from initial administration, we measured body weights to evaluate the toxicity of each adjuvant.

\section{Immune response against $\mathrm{CH}-\mathrm{HG}$ containing OVA + GM-CSF} Fifty microliters of $\mathrm{CH}-\mathrm{HG}, \mathrm{CFA}$, and IFA containing OVA + GM-CSF were injected subcutaneously into mice, and the adjuvants were boosted at 7 days using the same injection volume. The IgG, IgG1, and IgG2a levels in serum were measured by ELISA 3 weeks after the first immunization. Briefly, the presence of OVA-specific antibodies in the sera from $\mathrm{CH}-\mathrm{HG}$ mediated immunization of C57BL/6 mice (five per group) was determined by ELISA using microwell plates coated with OVA protein. Purified OVA protein was diluted to $0.5 \mu \mathrm{g} / \mathrm{ml}$ in PBS buffer $(\mathrm{pH} 7.4)$, and $50 \mu \mathrm{l}$ of that solution was then added to each well of 96-well microtiter plates. Purified OVA protein was used as a negative control. The plates were incubated overnight at $37^{\circ} \mathrm{C}$, followed by three washes with $300 \mu \mathrm{l}$ of PBS. After washing, $200 \mu \mathrm{l}$ of blocking solution ( $1 \%$ skim milk) was incubated at $37^{\circ} \mathrm{C}$ for $1 \mathrm{hr}$, and then the plates were washed three additional times using PBS containing $0.05 \%$ Tween 20 (PBS-T). Serial dilutions of the tested sera were made $(0.1 \mathrm{ml} /$ well $)$, and the plates were incubated for $1 \mathrm{hr}$ at $37^{\circ} \mathrm{C}$. The plates were washed with PBS-T and incubated with $0.1 \mathrm{ml}$ of alkaline phosphataseconjugated rabbit anti-mouse antibodies (Zymed) per well for $1 \mathrm{hr}$ at $37^{\circ} \mathrm{C}$. The plates were again washed with PBS$\mathrm{T}$ and incubated with alkaline phosphatase substrate (100 $\mu \mathrm{l} /$ well, Sigma) according to the manufacturer's instructions for $1 \mathrm{hr}$ at $37^{\circ} \mathrm{C}$. Plates were read on a MicroElisa reader at a wavelength of $650 \mathrm{~nm}$ (Additional file 1). 


\section{Flow cytometry analysis}

To assess OVA specific immune responses, we immunized mice with $50 \mu \mathrm{l}$ of one of five different solutions; 1) $50 \mu \mathrm{g}$ of OVA solution, 2) $50 \mu \mathrm{g}$ of OVA solution with GM-CSF, 3) CH-HG containing $50 \mu \mathrm{g}$ of OVA, 4) $\mathrm{CH}-$ HG containing $50 \mu \mathrm{g}$ of OVA $+50 \mathrm{ng}$ of GM-CSF, or 5) IFA containing $50 \mu \mathrm{g}$ of OVA $+50 \mathrm{ng}$ of GM-CSF. Splenocytes were harvested from the immunized mice (five per group) for 2 weeks after the first immunization. Prior to intracellular cytokine staining, $5 \times 10^{6}$ pooled splenocytes from each immunization group were incubated overnight with $1 \mu \mathrm{g} / \mathrm{ml}$ OVA peptide containing MHC class I epitope (aa 257-264) or MHC class II epitope (aa 323-339) to detect OVA-specific $\mathrm{CD}^{+}$and $\mathrm{CD}^{+} \mathrm{T}$ cell precursors. Intracellular IFN- $\gamma$ and IL4 staining, as well as flow cytometric analysis were performed using a Becton-Dickinson FACScan with CELLQuest software (Becton Dickinson Immunocytometry Systems, Mountain View, CA). The number of OVAspecific INF- $\gamma$ or IL4 secreting CD4 ${ }^{+} \mathrm{T}$ cells and INF- $\gamma$ secreting $\mathrm{CD}^{+} \mathrm{T}$ cells were assessed by intracellular cytokine staining and FACScan analysis.

\section{Statistical analysis}

Differences in continuous variables were analyzed by Student's $t$-test for comparing two groups and ANOVA was performed to compare differences between multiple groups. For values that were not normally distributed, the MannWhitney rank sum test was used. The statistical package for the Social Sciences (SPSS, Inc.) was used for all statistical analyses. A $p$ value of $<0.05$ was considered statistically significant.

\section{Results}

\section{CH-HG safety after SC injection in mice}

We first confirmed hydrogel formation after SC injection of $\mathrm{CH}$ into mice (Figure 1A). The $\mathrm{CH}$ solution displayed a liquid-solid phase transition in a temperature-dependent manner and formed an endothermic hydrogel at the injection site. Furthermore, we noted no pus, discharge, or scab formation at the injection site of CH-HG solutions, indicating improved safety compared to commercially available CFA or IFA (Figure 1A). Moreover, $\mathrm{CH}-\mathrm{HG}$ was not present at the injection site 2 weeks after injection, suggesting increased biodegradation compared to CFA and IFA (Figure 1B). We also checked the body weights of mice for 2 weeks after SC injection of $\mathrm{CH}-\mathrm{HG}$ to evaluate adjuvant toxicity (Figure $1 \mathrm{C}$ ). While CFA and IFA injected groups weighted less, the $\mathrm{CH}-\mathrm{HG}$ injected group did not weigh significantly different than the control group, suggesting that the $\mathrm{CH}-\mathrm{HG}$ system displayed enhanced safety at the in injection site. Therefore, CH-HG may be useful as a safer adjuvant system in mice.

\section{CH-HG containing OVA and GM-CSF induced titers of OVA specific lgG}

To determine the effectiveness of CH-HG in inducing an immune response, we assessed the humoral immune
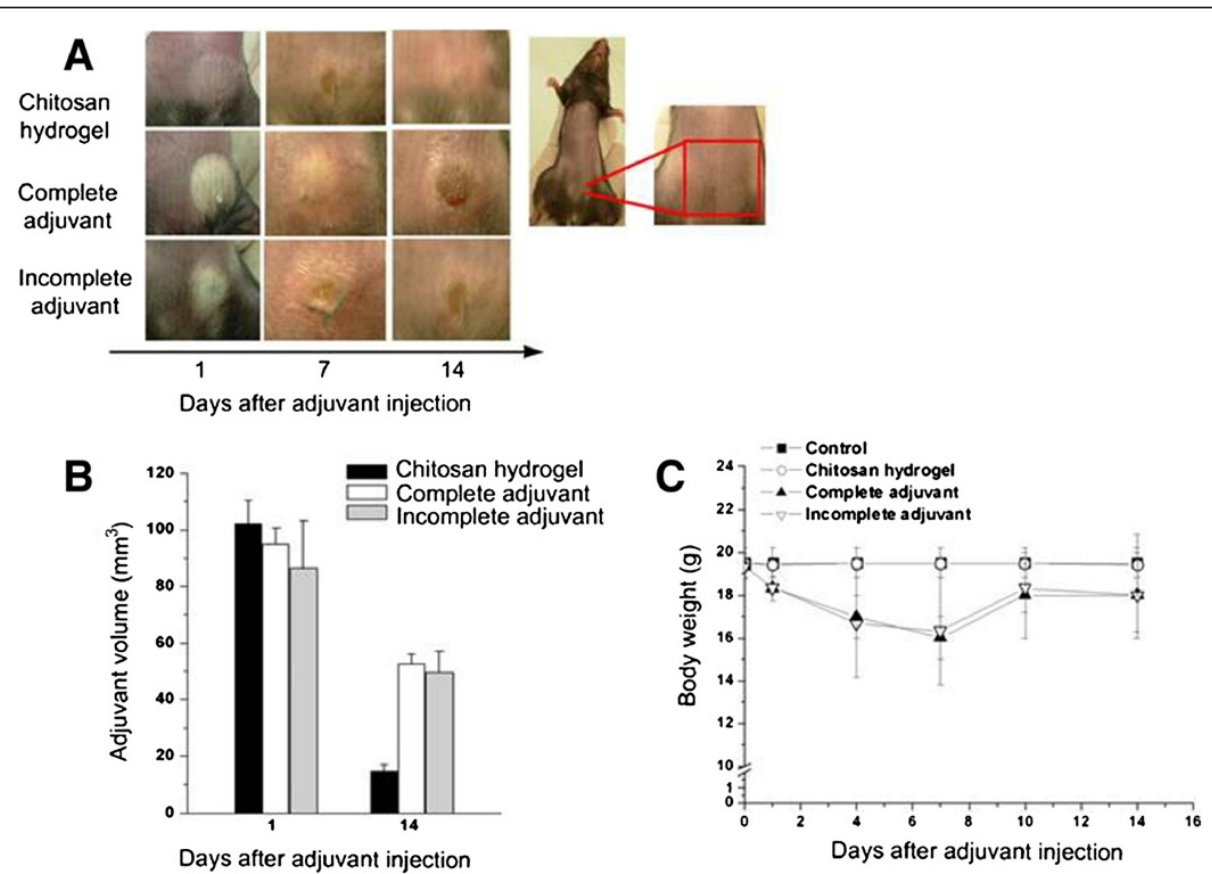

Figure 1 Safety of CH-HG compared to that of commercial adjuvants, in mice after subcutaneous administration. (A) Morphology of the injection site in mice. (B) Change in volume of injected adjuvants after 14 days. (C) Body weight of mice. The data are presented as the means \pm S.D. $(n=5)$. 
response to $\mathrm{CH}-\mathrm{HG}$ containing OVA (CH-HG/OVA). Two weeks after the last SC injection of CH-HG/OVA into mice, OVA specific IgG expression was significantly induced, and measured 10-fold higher than that in mice immunized with soluble OVA alone $(p<0.001)$ (Figure 2A). Additionally, OVA specific IgG progressively increased rising to a $10^{7}$-fold increase in expression 5 weeks after the last immunization (Additional file 2: Figure S1). Based on the immune response data for $\mathrm{CH}-\mathrm{HG} / \mathrm{OVA}$, we next tested immune responses for $\mathrm{CH}-\mathrm{HG} / \mathrm{OVA}$ with $50 \mathrm{ng}$ of GM-CSF (CH-HG/OVA + GM-CSF), which further increased the IgG titer $\left(10^{3}\right.$-fold increase) compared to the CH-HG/OVA without GM-CSF $(p<0.001)$. We therefore chose CH-HG/OVA + GM-CSF for all subsequent experiments (Figure 2A). In addition, there were higher titers of OVA-specific IgG1 than of IgG2a in serum from mice immunized with $\mathrm{CH}-\mathrm{HG} / \mathrm{OVA}+$ GM-CSF (Figure 2B). These data demonstrate that immunization with $\mathrm{CH}-\mathrm{HG} / \mathrm{OVA}+\mathrm{GM}-\mathrm{CSF}$ significantly enhances OVA-specific humoral immune response (IgG1), and the $\mathrm{CH}-\mathrm{HG}$ induced immune response would be strongly dependent on the Th2 type immune response.

\section{CH-HG/OVA + GM-CSF induction of OVA-specific IgG compared to CFA and IFA}

Next, we checked whether $\mathrm{CH}-\mathrm{HG}$ could increase OVA specific IgG expression compared to commercialized CFA or IFA. We first measured OVA specific IgG from the serum of mice immunized with $\mathrm{CH}-\mathrm{HG} / \mathrm{OVA}+$ GM-CSF, CFA/OVA + GM-CSF, or IFA/OVA + GM-CSF (Figure 3A). Mice immunized with $\mathrm{CH}-\mathrm{HG} / \mathrm{OVA}+\mathrm{GM}$ CSF generated significantly higher titers of OVA-specific antibody responses compared to mice immunized with CFA/OVA + GM-CSF or IFA/OVA + GM-CSF $(p<0.01)$. Furthermore, the sera of mice immunized with $\mathrm{CH}-\mathrm{HG} /$ OVA + GM-CSF had significantly higher titers of OVAspecific IgG1 antibodies compared to mice immunized with CFA/OVA + GM-CSF or IFA/OVA + GM-CSF (Figure $3 \mathrm{~B}$ ). These data indicate that immunization with $\mathrm{CH}-\mathrm{HG} / \mathrm{OVA}+\mathrm{GM}-\mathrm{CSF}$ elicits a stronger OVAspecific humoral immune response, providing a potential alternative in clinical practice to existing commercially available adjuvants such as CFA or IFA. However, the IgG2a titers were similar between all groups of mice (Figure 3C), which suggests that CH-HG/OVA + GM-CSF may not contribute the IgG2 mediated immune responses.

\section{CH-HG/OVA + GM-CSF generated an enhanced OVA-specific cellular immune response}

$\mathrm{T}$ cell mediated immunity is important for controlling tumor cells and intracellular infections caused by bacteria or viruses. For a quantitative characterization of OVAspecific $\mathrm{CD}^{+} \mathrm{T}$ cell-mediated immune responses, we performed flow cytometry analysis with or without the MHC class II-restricted peptide $\mathrm{OVA}_{323-339}$ (ISQAV HAAHAEINEAGR). As shown in Figure 4A and 4B, the
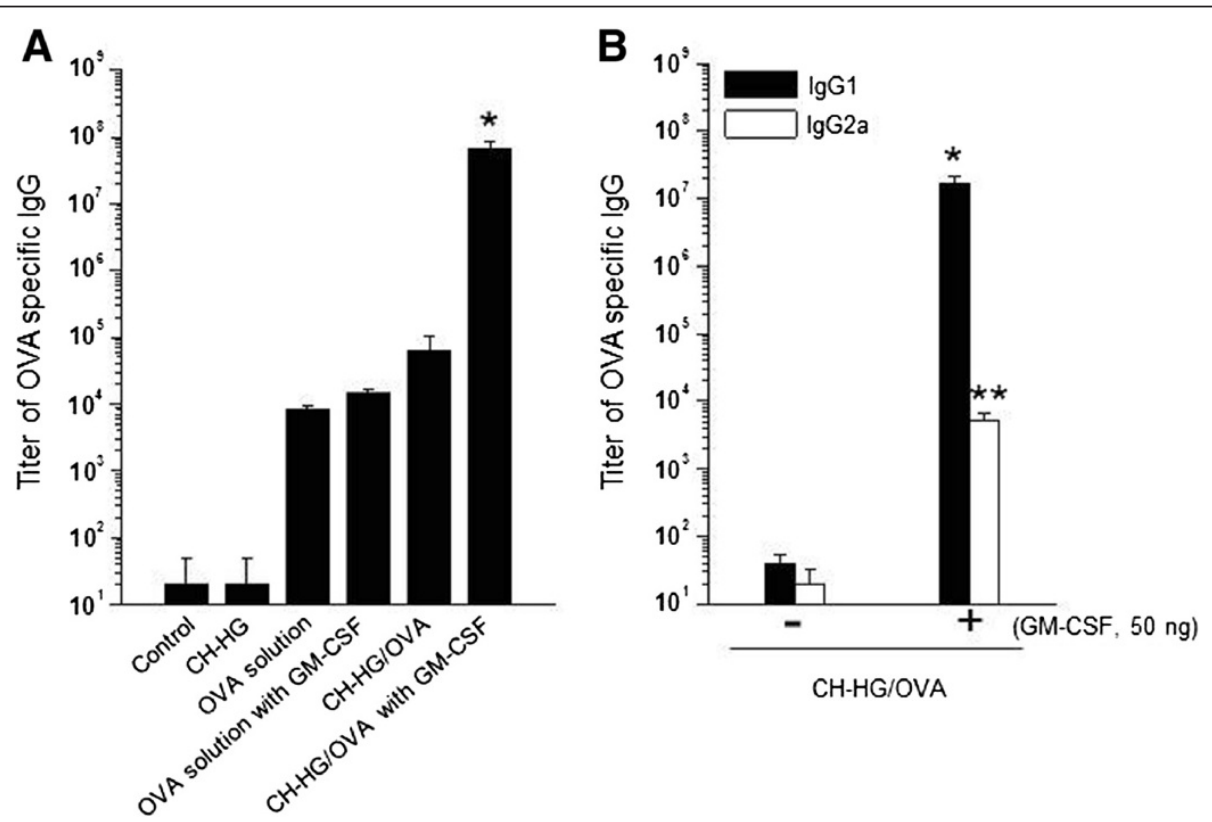

Figure 2 OVA-specific immune responses in mice immunized with $50 \mu \mathrm{g}$ of OVA solution, $\mathrm{CH}-\mathrm{HG}$ containing $50 \mu \mathrm{g}$ of $\mathrm{OVA}$, and $\mathrm{CH}-\mathrm{HG}$ containing $\mathbf{5 0} \mathbf{\mu g}$ of OVA and $\mathbf{5 0} \mathbf{n g}$ of GM-CSF. Sera were collected from immunized mice (3 per group) 2 weeks after the last immunization and were used to characterize OVA-specific antibodies. Mice received an immunization with $50 \mu \mathrm{g}$ of OVA per mouse via subcutaneous injection. Seven days after immunization, experimental groups were boosted. (A) Titers of OVA specific lgG. (B) Titers of OVA specific lgG for humoral or cellular immune response. The data are presented as the means \pm S.D. $(n=5),\left({ }^{*} p<0.001,\left({ }^{* *} p<0.002\right)\right.$. 


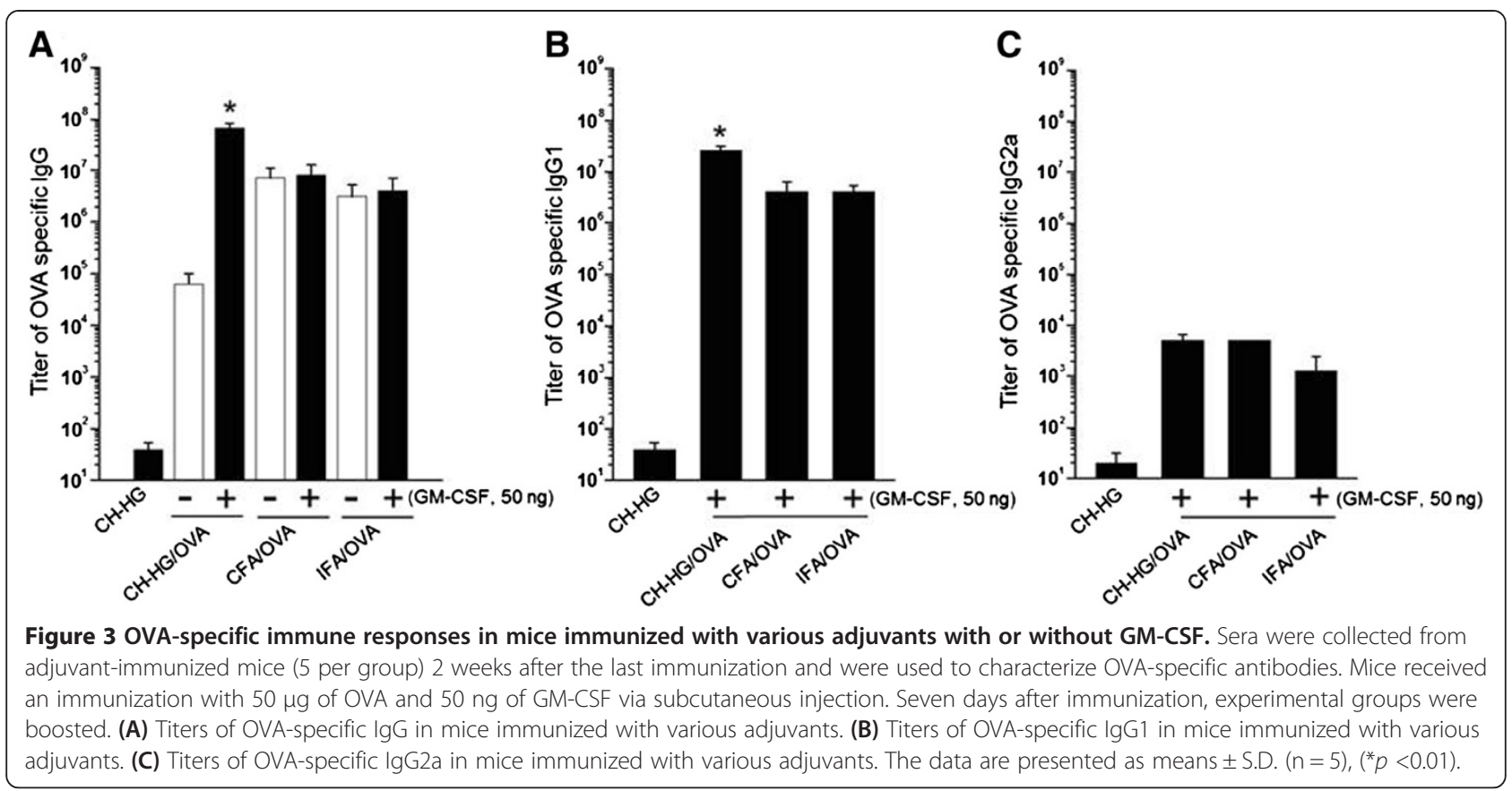

numbers of both OVA-specific Th1 $\mathrm{CD}^{+}$and Th2 $\mathrm{CD}^{+}$ $\mathrm{T}$ cells in the splenocytes of $\mathrm{CH}-\mathrm{HG} / \mathrm{OVA}+\mathrm{GM}-\mathrm{CSF}$ immunized mice were significantly increased compared to that of mice immunized with OVA or $\mathrm{CH}-\mathrm{HG} / \mathrm{OVA}$ $(p<0.001)$. We further measured $\mathrm{CD}^{+} \mathrm{T}$ cell immune responses by performing intracellular cytokine staining with or without $\mathrm{MHC}$ class I-restricted peptide $\mathrm{OVA}_{257-264}$
(SIINFEKL). As shown in Figure 4C, the numbers of OVA-specific $\mathrm{CD}^{+} \mathrm{T}$ cells in splenocytes of mice immunized with $\mathrm{CH}-\mathrm{HG} / \mathrm{OVA}+\mathrm{GM}-\mathrm{CSF}$ were higher than those of the other groups $(p<0.001)$. Collectively, these data demonstrate that immunization with $\mathrm{CH}-\mathrm{HG} / \mathrm{OVA}$ + GM-CSF adjuvant could elicit antigen-specific cellular immune responses.

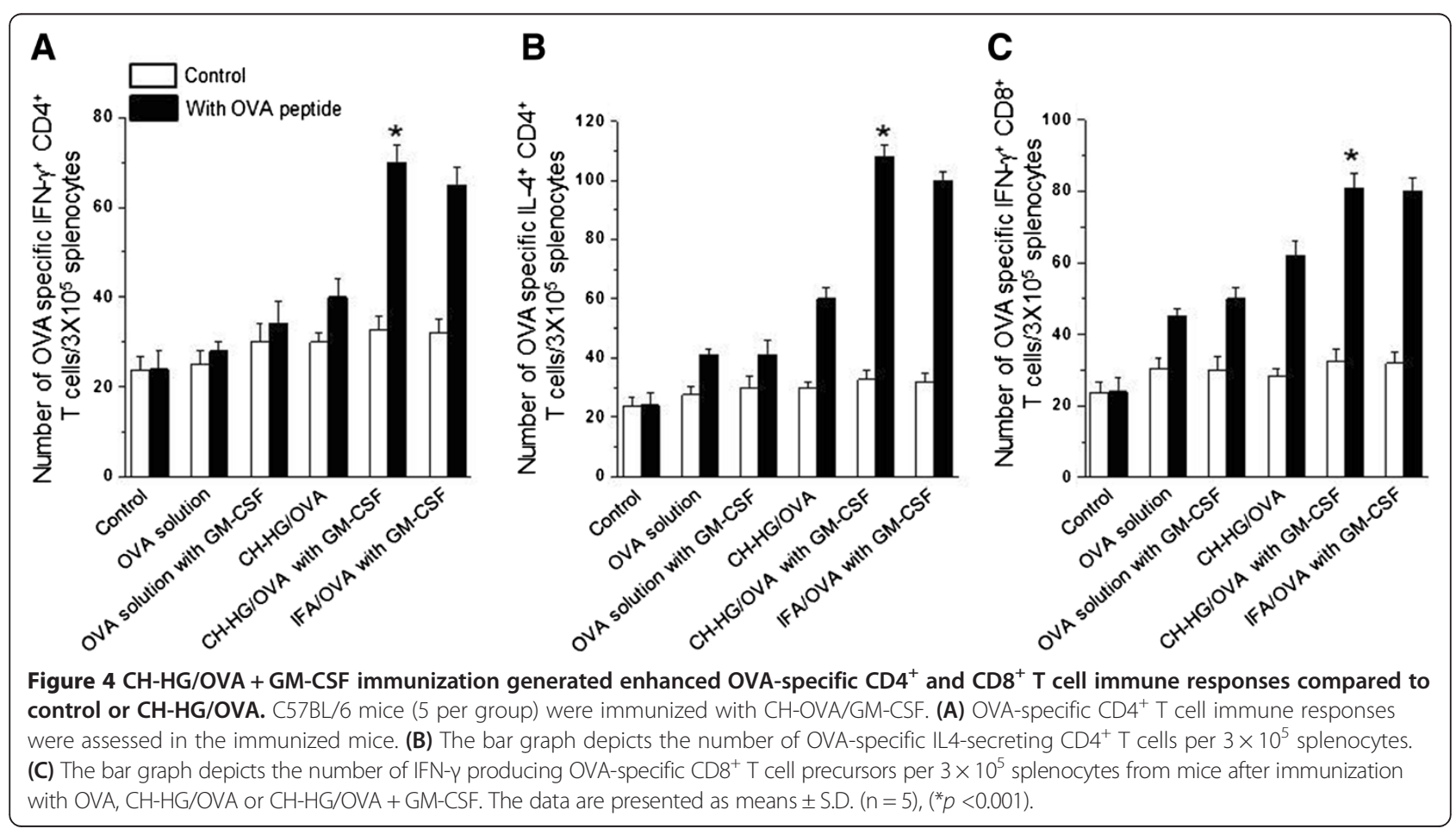




\section{Discussion}

Adjuvant-based vaccination in immunotherapy is highly effective, but it is associated with inherent safety and toxicity problems that need to be overcome before use in clinical settings $[3,4]$. We demonstrate here that a novel $\mathrm{CH}$-HG based adjuvant system loaded with GMCSF, a broad immune-modulating cytokine, led to potent both humoral and cellular immune responses without severe side effects. This approach has broad utility for adjuvant mediated therapeutic materials as well as other immunotherapy applications.

Several synthetic biomaterials have been proposed as adjuvants for effective antigen delivery. These include microparticles [18], nanofibers [19], metal-based particles [20], and emulsions [21]. Although many types of compounds can be potentially used as delivery agents, their safety and evoked immune responses cause concerns. The development of adjuvant-based vaccination for immunotherapy, therefore, requires clinically suitable, safe, and effective delivery systems.

Therapeutic materials packaged into $\mathrm{CH}-\mathrm{HG}$ may provide a clinically viable approach for the development of a vaccine related immunotherapy. The biocompatibility and biodegradability of these systems are key parameters for medical and pharmaceutical applications [9]. Here, we demonstrate that a novel hydrogel-based adjuvant system loaded with OVA and GM-CSF led to potent immune responses in mice. This study implements a novel adjuvant with improved safety and reduced toxicity. In addition, the CH-HG system allows for the co-delivery of therapeutic materials, such as an antigen, which could further enhance the antigen specific immune response without increasing toxicity. Injection of $\mathrm{CH}-\mathrm{HG}$ is simple because $\mathrm{CH}-\mathrm{HG}$ is a liquid phase at room temperature and therefore, does not require surgery for insertion at the site of the disease, which would disrupt the disease environment. Moreover, the biodegradation rate of injected hydrogel can be controlled by modifying the injected volume. In this study, we injected $50 \mu \mathrm{l}$ of chitosan solution, which degrades in approximately 2 weeks, suggesting that this amount can be used for repeated injections. This local depot system may be attractive for many biomedical applications, including administration of anesthetic agents after surgery and treatment of certain skin, breast, or neck cancers. Such an approach may also be useful for adjuvant therapy, or as a local treatment for chronic periodontitis. Although the $\mathrm{CH}-\mathrm{HG}$ mediated adjuvant platform can be useful for diseases associated with the immune system and with the intent to enhance immune response, additional possibilities of optimized loading for effective cytokine or immune modulator using the $\mathrm{CH}-\mathrm{HG}$ platform may be explored and developed for research purposes.

\section{Conclusion}

In summary, we have developed a novel adjuvant system that yields high immune responses without negative side effects at the local site of interest due to matrix toxicity. Our data demonstrate that $\mathrm{CH}-\mathrm{HG}$ based local antigen delivery can invoke antigen specific $\mathrm{CD} 8^{+} \mathrm{T}$ cell immunity. Such $\mathrm{CH}-\mathrm{HG}$ based adjuvant strategies may have broad potential as antigen delivery platforms in human disease and represent an opportunity for further development of vaccine based immunotherapeutics.

\section{Additional files}

\section{Additional file 1: Arrive guidelines checklist.}

Additional file 2: Figure S1. OVA-specific humoral immune responses in mice immunized with $\mathrm{CH}-\mathrm{HG} / \mathrm{OVA}+\mathrm{GM}-\mathrm{CSF}$ over time. Sera were collected from CH-HG/OVA + GM-CSF-immunized mice (5 per group) for 2 weeks after the last immunization and were used to characterize OVA-specific antibodies. Mice received an immunization with $50 \mu \mathrm{g}$ of OVA and 50 ng of GM-CSF per mouse via subcutaneous injection. The data are presented as means \pm S.D. $(n=5)$.

\section{Competing interests}

The authors declare that they have no competing interests.

\section{Authors' contributions}

KHN and YMP performed the preparation and characterization of the hydrogel. HSK, THK, KHS, YHL, YB, and HNJ participated in the animal experiment and $\mathrm{T}$ cell immune response. IDJ, BCS, KML, and SYS conceive of the study and participated in data analysis. $\mathrm{HDH}$, and TWK participated in its design and coordination. All authors read and approved the final manuscript.

\section{Acknowledgments}

This work was supported by the National Research Foundation of Korea (NRF) grant funded the Korea government (NRF-2012R1A2A1A03008433) (Y.M.P.), (NRF-2013M3A9D3045881), and (NRF-2012R1A2A2A01007527). This work was supported by Basic Research Laboratory Program through the National Research Foundation of Korea (NRF) funded by the Ministry of Science, ICT \& Future Planning (No.2013R1A4A1069575) (H.D.H.). This work was supported by a grant of the Korea Healthcare technology R\&D Project, Ministry of Health and Welfare, Republic of Korea (A062260) (T.W.K.).

\section{Author details}

${ }^{1}$ Laboratory of Infection and Immunology, Graduate School of Medicine, Korea University, Gojan-1 Dong, Ansan-Si, Gyeonggi-Do 425-707, South Korea. ${ }^{2}$ Department of Biochemistry, College of Medicine, Korea University, Seoul, Korea. ${ }^{3}$ Department of Immunology, School of Medicine, Konkuk University, 268 Chungwondaero, Chungju-Si, Chungcheongbuk-Do 380-701, South Korea. ${ }^{4}$ Department of Immunology and Physiology and Functional Genomics Institute, College of Medicine, Konkuk University, 268

Chungwondaero, Chungju-Si, Chungcheongbuk-Do 380-701, South Korea. ${ }^{5}$ Research Center for Medicinal Chemistry, Division of Drug Discovery Research, Korea Research Institute of Chemical Technology, 141 Gajeong-roYuseong-gu, Daejeon 305-600, South Korea. ${ }^{6}$ Department of Microbiology and Immunology, College of Medicine, Seoul National University, 28 Yongon-dongJongno-gu, Seoul 110-799, Republic of Korea. ${ }^{7}$ Department of Biomedical Sciences, College of Medicine, Seoul National University, 28 Yongon-dongJongno-gu, Seoul 110-799, Republic of Korea.

Received: 19 May 2014 Accepted: 10 October 2014

Published online: 18 October 2014

\section{References}

1. Wilson-Welder JH, Torres MP, Kipper MJ, Mallapragada SK, Wannemuehler MJ, Narasimhan B: Vaccine adjuvants: current challenges and future approaches. J Pharm Sci 2009, 98(4):1278-1316. 
2. Riese P, Schulze K, Ebensen T, Prochnow B, Guzman CA: Vaccine adjuvants: key tools for innovative vaccine design. Curr Top Med Chem 2013, 13(20):2562-2580.

3. Reed SG, Orr MT, Fox CB: Key roles of adjuvants in modern vaccines. Nat Med 2013, 19(12):1597-1608.

4. Chapel HM, August PJ: Report of nine cases of accidental injury to man with Freund's complete adjuvant. Clin Exp Immunol 1976, 24(3):538-541.

5. Bijker MS, van den Eeden SJ, Franken KL, Melief CJ, Offringa R, van der Burg SH: CD8+ CTL priming by exact peptide epitopes in incomplete Freund's adjuvant induces a vanishing CTL response, whereas long peptides induce sustained CTL reactivity. J Immunol 2007, 179(8):5033-5040.

6. Stewart-Tull DE: Freund's complete and incomplete adjuvants, preparation, and quality control standards for experimental laboratory animals use. Methods Mol Biol 2010, 626:59-72.

7. Seo SH, Han HD, Noh KH, Kim TW, Son SW: Chitosan hydrogel containing GMCSF and a cancer drug exerts synergistic anti-tumor effects via the induction of CD8+ T cell-mediated anti-tumor immunity. Clin Exp Metastasis 2009, 26(3):179-187.

8. Han HD, Mora EM, Roh JW, Nishimura M, Lee SJ, Stone RL, Bar-Eli M, Lopez-Berestein G, Sood AK: Chitosan hydrogel for localized gene silencing. Cancer Biol Ther 2011, 11(9):839-845.

9. Han HD, Nam DE, Seo DH, Kim TW, Shin BC: Preparation and biodegradation of thermosensitive chitosan hydrogel as a function of $\mathrm{pH}$ and temperature. Macromol Res 2004, 12:507-511.

10. Zhang C, Wang B, Wang M: GM-CSF and IL-2 as adjuvant enhance the immune effect of protein vaccine against foot-and-mouth disease. Virol J 2011, 8:7.

11. Ullenhag GJ, Frodin JE, Strigard K, Mellstedt H, Magnusson CG: Induction of IgG subclass responses in colorectal carcinoma patients vaccinated with recombinant carcinoembryonic antigen. Cancer Res 2002, 62(5):1364-1369.

12. Disis ML, Bernhard H, Shiota FM, Hand SL, Gralow JR, Huseby ES, Gillis S, Cheever MA: Granulocyte-macrophage colony-stimulating factor: an effective adjuvant for protein and peptide-based vaccines. Blood 1996, 88(1):202-210.

13. Morrissey PJ, Bressler L, Park LS, Alpert A, Gillis S: Granulocyte-macrophage colony-stimulating factor augments the primary antibody response by enhancing the function of antigen-presenting cells. J Immunol 1987, 139(4):1113-1119.

14. Sun X, Hodge LM, Jones HP, Tabor L, Simecka JW: Co-expression of granulocyte-macrophage colony-stimulating factor with antigen enhances humoral and tumor immunity after DNA vaccination. Vaccine 2002, 20(9-10):1466-1474.

15. Toubaji A, Hill S, Terabe M, Qian J, Floyd T, Simpson RM, Berzofsky JA, Khleif SN: The combination of GM-CSF and IL-2 as local adjuvant shows synergy in enhancing peptide vaccines and provides long term tumor protection. Vaccine 2007, 25(31):5882-5891.

16. Reid CD, Stackpoole A, Meager A, Tikerpae J: Interactions of tumor necrosis factor with granulocyte-macrophage colony-stimulating factor and other cytokines in the regulation of dendritic cell growth in vitro from early bipotent $\mathrm{CD} 34+$ progenitors in human bone marrow. J Immunol 1992, 149(8):2681-2688.

17. Golumbek PT, Azhari R, Jaffee EM, Levitsky HI, Lazenby A, Leong K, Pardoll DM: Controlled release, biodegradable cytokine depots: a new approach in cancer vaccine design. Cancer Res 1993, 53(24):5841-5844.

18. Igartua M, Hernandez RM, Esquisabel A, Gascon AR, Calvo MB, Pedraz JL: Enhanced immune response after subcutaneous and oral immunization with biodegradable PLGA microspheres. J Control Release 1998, 56(1-3):63-73.

19. Chesson CB, Huelsmann EJ, Lacek AT, Kohlhapp FJ, Webb MF, Nabatiyan A, Zloza A, Rudra JS: Antigenic peptide nanofibers elicit adjuvant-free CD8 (+) T cell responses. Vaccine 2014, 32(10):1174-1180.

20. Xu Y, Tang H, Liu JH, Wang H, Liu Y: Evaluation of the adjuvant effect of silver nanoparticles both in vitro and in vivo. Toxicol Lett 2013, 219(1):42-48.

21. Vono M, Taccone M, Caccin P, Gallotta M, Donvito G, Falzoni S, Palmieri E, Pallaoro M, Rappuoli R, Di Virgilio F, De Gregorio E, Montecucco C, Seubert A: The adjuvant MF59 induces ATP release from muscle that potentiates response to vaccination. Proc Natl Acad Sci U S A 2013, 110(52):21095-21100

\section{doi:10.1186/s12865-014-0048-x}

Cite this article as: Noh et al: GM-CSF-loaded chitosan hydrogel as an immunoadjuvant enhances antigen-specific immune responses with reduced toxicity. BMC Immunology 2014 15:48.

\section{Submit your next manuscript to BioMed Central and take full advantage of:}

- Convenient online submission

- Thorough peer review

- No space constraints or color figure charges

- Immediate publication on acceptance

- Inclusion in PubMed, CAS, Scopus and Google Scholar

- Research which is freely available for redistribution 